\title{
Fórmulas de selección económica de contratistas en adjudicación de obras de infraestructura vial: Estudio de caso Valle del Cauca, Colombia $^{1}$
}

\author{
Economic's selection formula of contractors in adjudication for \\ road infrastructure works: case study Valle del Cauca, Colombia
}

\section{Fórmula econômica de seleção de empreiteiros em adjudicação de obras de infraestrutura viária: estudo de caso Valle del Cauca, Colômbia}

\author{
N. A. Zambrano, M. A. Jaramillo, D. D. Pérez y M. F. Serrano \\ Recibido: septiembre 2 de 2017 - Aceptado: junio 30 de 2018
}

\begin{abstract}
Resumen- El estatuto general para la contratación de la administración pública en Colombia (Ley 80 de 1983) establece que las entidades estatales (Ley 489 de 1988), excepto aquellas en las cuales la ley no les obliga a solicitar más de una propuesta, deben seleccionar a sus contratistas a través de licitaciones, convocatorias o concurso público, o mediante cualquier modalidad contractual, según el régimen jurídico de contratación
\end{abstract}

${ }^{1}$ Producto derivado del trabajo de grado de maestría "Análisis de los requerimientos técnicos, financieros, organizacionales y de experiencia en proyectos de infraestructura vial: caso valle del cauca 2010-2015" Dirigido por el Grupo de Investigación DeCoR, de la Pontificia Universidad Javeriana Cali y la Universidad Pontificia Bolivariana Bucaramanga. 2016

N. A. Zambrano, Pontificia Universidad Javeriana Cali, Colombia, email: nikolas.zambrano@javerianacali.edu.co.

M. A. Jaramillo, Pontificia Universidad Javeriana, Cali, Colombia, email: maria.jaramillog@javerianacali.edu.co.

D. D. Pérez, Pontificia Universidad Javeriana, Cali, Colombia, email: ddperez@javerianacali.edu.co.

M. F. Serrano, Pontificia Universidad Javeriana Cali, Colombia, email: maria.serrano@javerianacali.edu.co, autor responsable de correspondencia.

Como citar este artículo: Zambrano, N.A., Jaramillo, M. A. Pérez, D. D. y Serrano, M. F. Fórmulas de selección económica de contratistas en adjudicación de obras de infraestructura vial: Estudio de caso Valle del Cauca, Colombia, Entre Ciencia e Ingeniería, vol. 12, no. 24, pp.60-67, julio-diciembre, 2018. DOI: http://dx.doi.org/10.31908/19098367.3810

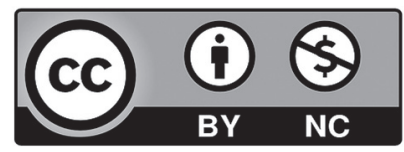

Attribution-NonCommercial 4.0 International (CC BY-NC 4.0) que le sea aplicable (Ley 816 de 2003). Con el fin de garantizar la transparencia en los procesos licitatorios, se han planteado diferentes tipos de fórmulas $y$, aun así, en ocasiones termina favoreciéndose el mismo grupo de personas. En este artículo se analiza el impacto de la fórmula de selección de oferentes en proyectos de infraestructura vial en el Departamento del Valle del Cauca. Con este propósito, se tomaron como base pliegos de condiciones de contratos disponibles en la plataforma digital del Servicio Electrónico de Contratación Pública, SECOP. Como resultado, se encontró que las fórmulas más empleadas son la media aritmética y la media geométrica con presupuesto oficial. Se deja entrever que, a pesar de la gran variedad de fórmulas, se presenta una alta adjudicación de obras de manera simultánea a personas naturales participando en diferentes modalidades de asociación.

Palabras clave- acuerdos económicos, administración pública, finanzas, formulación de proyectos, obras públicas.

Abstract - The general statute for contracting in public administration in Colombia (Law 80 of 1983) establishes that state entities (Law 489 of 1988), except those that the law does not require more than one proposal, must select their contractors through: public tenders, invitations or any contractual modality, according to the legal regime applicable to the contract (Law 816 of 2003). In order to guarantee transparency in the bidding processes, have been proposed different types of formulas and even then, they sometimes end up favoring the same group of people. This article analyzes the impact of the bidder selection formula on road infrastructure projects in the Department of Valle del Cauca. For this purpose, contract specifications were available based on the digital platform of the Electronic Procurement Service SECOP. 
As a result, it was found that the most used formulas are the arithmetic mean and the geometric mean with official budget. It is clear that, despite the great variety of formulas, there is a high works simultaneously adjudicated to natural persons participating in different forms of association

Keywords - economic accords, public administration, public works, finances, project selection.

Resumo - A administração na Colômbia (Lei 80 de 1983) estabelece que as entidades estatais (Lei 489 de 1988), exceto aquelas em que a lei não exige mais de uma proposta, devem selecionar seus contratados através de licitações públicas, convites ou qualquer modalidade contratual, de acordo com o regime jurídico aplicável ao contrato (Lei 816 de 2003). Para garantir a transparência nos processos de licitação, foram propostos diferentes tipos de fórmulas e, mesmo assim, acabam por favorecer o mesmo grupo de pessoas. Este artigo analisa o impacto da fórmula de seleção de licitante em projetos de infraestrutura rodoviária no Departamento do Valle del Cauca. Para isso, as especificações do contrato estavam disponíveis com base na plataforma digital do Serviço de Compras Eletrônicas SECOP. Como resultado, verificou-se que as fórmulas mais utilizadas são a média aritmética e a média geométrica com o orçamento oficial. É claro que, apesar da grande variedade de fórmulas, há um alto trabalho simultaneamente adjudicado a pessoas físicas que participam de diferentes formas de associação

Palavra chave-acordos econômicos, administração pública, obras públicas, finanças, seleção de projetos.

\section{INTRODUCCIÓN}

$\mathrm{E}_{\text {resher }}^{\mathrm{L}}$ desarrollo de las comunidades de un país está relacionado con la adecuada planeación del crecimiento mediante la ejecución de diferentes obras, lo que a su vez depende del buen uso que se dé a los fondos destinados para suplir las necesidades insatisfechas identificadas previamente. Según esto, un diagnóstico actualizado de necesidades reales de las comunidades debería ser un requisito para la asignación de partidas presupuestales por parte del Estado.

Para el caso de Colombia, estos diagnósticos deberían estar incluidos en los planes de desarrollo de cada municipio, documentos en los cuales se identifican las obras prioritarias [1] a corto, mediano y largo plazo, y con los que se busca alcanzar el objetivo universal de igualdad de condiciones que promueve el capitalismo como promesa modernizadora [2].

Partiendo de esta línea base, se inicia un proceso de contratación de bienes y servicios públicos que debe regirse por los principios de la transparencia, equidad y justicia.

Lamentablemente, por diferentes motivos, en ocasiones algunos procesos de adjudicación generan la sospecha de corrupción, bien sea porque se repiten los mismos contratistas bajo diferentes modalidades contractuales o porque las obras se inician y no se concluyen, o se inician y presentan un comportamiento contractual que termina en obras inconclusas sin sanciones para los responsables de estos hechos, realidades que debilitan la cohesión social y reducen la posibilidad de construir un pacto social sólido entre la población [3].
En la actualidad, el Estado colombiano cuenta con el Sistema Electrónico de Contratación Pública (SECOP), herramienta de consulta disponible al público, a través de la cual se puede acceder a la información relacionada con los contratos y los procesos de selección de contratistas [4]. De esta forma, los ciudadanos pueden hacer seguimiento y llevar a cabo la trazabilidad de los recursos que se han otorgado para el desarrollo de las distintas obras [1].

En este artículo se realiza un análisis de fórmulas empleadas en los procesos licitatorios y la posible incidencia de la fórmula en la adjudicación de contratistas. El estudio se realizó en el Valle del Cauca para el periodo 2010 a 2015 en el sector vial, por cuanto justamente, es el sector en el que el gobierno actual ha realizado inversiones de gran importancia $y$, por ende, las partidas presupuestales asignadas, ejecutadas o no, tienen una fuerte repercusión en el presupuesto de la nación [5].

\section{Metodología}

La metodología de este estudio se basó en el análisis de hechos ya cumplidos en procesos licitatorios de obra pública. Para el desarrollo de la investigación se siguieron las siguientes etapas:

\section{A. Consulta de la información disponible en la página del Servicio Electrónico de Contratación, SECOP}

Se revisaron los pliegos de condiciones relacionados con proyectos de infraestructura vial en el departamento del Valle de Cauca, seleccionando aquellos proyectos del grupo G (Terrenos, edificios, estructuras y vías), Segmento: (95) Terrenos, edificios, estructuras y vías adjudicados bajo la modalidad de contratación por Licitación pública en el Valle del Cauca, entre el 2010 y el 2015, con partidas iguales o superiores a 350 millones de pesos.

\section{B. Selección de la muestra de estudio}

En el SECOP se identificaron 132 Licitaciones Públicas que cumplen con los criterios establecidos para la investigación. Partiendo de esta población, se procedió a determinar el tamaño de la muestra a estudiar, con base en la ec. (1).

$$
n=\frac{N * Z^{2} * p * q}{d^{2}(N-1)+Z^{2} * p * q}
$$

Donde:

$\mathrm{N}=132$ procesos de contratación

$\mathrm{p}=50 \%$ y q $=50 \%$

$\mathrm{d}=0.1$ correspondiente a un porcentaje del $90 \%$.

$\mathrm{Z}=1.645$

La aproximación de $\mathrm{n}$ fue de 50 casos.

Teniendo en cuenta la gran variedad de características de los municipios del Valle del Cauca, se procedió a determinar la composición de la muestra por conglomerados [6]. Para ello, se partió de la información encontrada en el censo del 2005 sobre la población total de los habitantes en el Valle del Cauca (4'052.535 habitantes), distribuidos en 42 municipios 
[7]. Con esta información, se determinó la cantidad de procesos a considerar en función del número de habitantes en cada municipio, como se muestra en la Tabla I.

\section{Identificación de las fórmulas de adjudicación de los procesos seleccionados}

Se procedió a leer los pliegos de condiciones de los procesos seleccionados, tomando de allí la información particular sobre el proceso de evaluación de las propuestas económicas. Si bien es cierto los pliegos de condiciones en las licitaciones señalan requisitos habilitantes, tales como la capacidad jurídica, experiencia, capacidad organizacional y capacidad residual; $\mathrm{y}$, otros puntuables, como factor económico, factor técnico, puntaje por estímulo a la industria nacional, puntaje por renuncia al anticipo, entre otros [5], el presente estudio se centró en el análisis del impacto de las fórmulas incluidas para la selección de contratistas en lo relacionado con el factor económico.

TABLA I

COMPOSICIÓN DE LA MUESTRA POR CONGLOMERADO

\begin{tabular}{|l|l|c|c|}
\hline \multicolumn{1}{|c|}{ Zona } & \multicolumn{1}{|c|}{ Municipio } & \% población según censo 2005 & Número de procesos a revisar \\
\hline Centro & $\begin{array}{l}\text { Tuluá, Buga, El Cerrito, Guacarí, Bugalagrande, } \\
\text { Ginebra, Trujillo, Andalucía, Calima, Darién, San } \\
\text { Pedro, Riofrío, Yotoco, Restrepo. }\end{array}$ & 13,06 & 9 \\
\hline Norte & $\begin{array}{l}\text { Cartago, Zarzal, Roldanillo, La Unión, Anserman- } \\
\text { uevo, Toro, Bolívar, Obando, La Victoria, Alcalá, El } \\
\text { Águila, El Dovio, El Cairo, Versalles, Argelia, Ulloa. }\end{array}$ & 8,98 & 19 \\
\hline Occidente & Buenaventura. & 8,00 & 3 \\
\hline Oriente & Sevilla, Caicedonia, & 1,77 & 4 \\
\hline Sur & $\begin{array}{l}\text { Cali, Palmira, Jamundí, Yumbo, Candelaria, Florida, } \\
\text { Pradera, La Cumbre, Vijes. }\end{array}$ & 68,22 & 15 \\
\hline
\end{tabular}

El análisis de los demás requisitos se incorporó en otro artículo: "Análisis de la contratación estatal en proyectos de infraestructura vial: caso Valle del Cauca 2010-2015", actualmente en revisión.

\section{RESULTADOS}

\section{A. Fórmulas encontradas}

Dentro de los criterios puntuables se encuentra que, en los procesos licitatorios, la oferta económica tiene un valor preponderante. En total se identificaron siete posibles fórmulas:

\section{Media aritmética (MA)}

En este caso, se realiza el promedio aritmético de aquellas propuestas presentadas por contratistas que cumplieron los requisitos habilitantes, haciendo uso de la ec. (2) [4]:

$$
X=\sum_{i=1}^{n} \frac{x_{i}}{n}
$$

Donde:

$\mathrm{X}=$ Media aritmética.

$\mathrm{x}_{\mathrm{i}}=$ Valor de la oferta i sin decimales.

$\mathrm{n}=$ Número total de ofertas válidas presentadas.

Una vez determinado el valor de la media aritmética, se realiza una ponderación de las ofertas presentadas para la obtención del puntaje mediante la ec. (3):

$$
\text { Puntaje } i=\left\{\begin{array}{c}
150 *\left(1-\left(\frac{X-V_{i}}{X}\right)\right), \text { valores menores o iguales a } X \\
150 *\left(1-2\left(\frac{\left\lfloor X-V_{i} \nmid\right.}{X}\right)\right), \text { valores mayores a } X
\end{array}\right.
$$

Donde:

$\mathrm{V}_{\mathrm{i}}=$ Valor total de cada una de las ofertas $\mathrm{i}$, sin decimales

\section{Media aritmética alta (MAA)}

En este caso, se calcula la media aritmética entre el valor total sin decimales de la oferta válida más alta y el promedio aritmético de las ofertas válidas (las que pasaron los requisitos habilitantes), y la asignación de puntos de la proximidad de las ofertas a dicha media aritmética, como resultado de aplicar la ec. (4) [4].

$$
X_{A}=\frac{V_{\max }+X}{2}
$$

Donde:

$\mathrm{X}_{\mathrm{A}}=$ Media aritmética alta.

$\mathrm{V}_{\max }=$ Valor total sin decimales de la oferta más alta.

$\mathrm{X}=$ Promedio aritmético de las ofertas válidas.

Una vez se ha obtenido la media aritmética alta, se procede a calcular la ponderación de las ofertas en conformidad con la ec. (5):

Puntaje $i=\left\{\begin{array}{c}150 *\left(1-\left(\frac{X_{A}-V_{i}}{X}\right)\right) \text { Para valores menores o iguales a } X_{A} \\ 150 *\left(1-2\left(\frac{\nmid X_{A}-V_{i} \nmid}{X}\right)\right) \text { Para valores mayores a } X_{A}\end{array}\right.$

Donde:

$\mathrm{X}_{\mathrm{A}}=$ Media aritmética alta.

$\mathrm{V}_{\mathrm{i}}=$ Valor total de cada una de las ofertas $\mathrm{i}$, sin decimales

$\mathrm{i}=$ Número de oferta. 
3. Media geométrica con presupuesto oficial (MGPO)

Para la aplicación de esta fórmula, se determina la media geométrica de los presupuestos incluidos en las ofertas que fueron válidas y el presupuesto oficial un número determinado de veces, y la asignación de puntos en función del acercamiento de las ofertas a dicha media geométrica.

Para definir el número de veces que se incluye el presupuesto oficial se tiene en cuenta lo establecido en la Tabla II [4]. Y sucesivamente, por cada tres (3) ofertas válidas se incluirá una vez más el presupuesto oficial en el cálculo del puntaje. La expresión para este cálculo se indica en la ec. (6):

$$
\begin{gathered}
G_{P O}=\sqrt[(n v+n)]{P O * P O * \ldots \ldots * P O_{n v} * P_{1} * \ldots \ldots * P_{n}} \\
\text { TABLA II. }
\end{gathered}
$$

ASIGNACIÓN DEL NÚMERO DE VECES (NV) DEL PRESUPUESTO OFICIAL

\begin{tabular}{|c|c|}
\hline Número de ofertas & $\begin{array}{c}\text { nv que se incluye el } \\
\text { presupuesto oficial }\end{array}$ \\
\hline $1-3$. & 1 \\
\hline $4-6$. & 2 \\
\hline $7-9$. & 3 \\
\hline $10-12$. & 4 \\
\hline $13-15$. & 5 \\
\hline$\ldots \ldots$ & \\
\hline
\end{tabular}

Donde:

$\mathrm{G}_{\mathrm{PO}}=$ Media geométrica con presupuesto oficial.

$\mathrm{nv}=$ Número de veces que se incluye en el presupuesto oficial (PO)

$\mathrm{n}=$ Número de ofertas válidas

$\mathrm{PO}=$ Presupuesto oficial del proceso de contratación.

$\mathrm{Pi}=$ Valor de la oferta económica del proponente, sin decimales

Para la asignación de puntaje de cada proponente se tiene en cuenta la ec. (7):

Puntaje $i=\left\{\begin{array}{c}150 *\left(1-\left(\frac{G_{p o}-V_{i}}{G_{p o}}\right)\right), \text { valores menores o iguales a } G_{p o} \\ 150 *\left(1-2\left(\frac{\nmid G_{p o}-V_{i} \nmid}{X G_{p o}}\right)\right), \text { valores mayores a } G_{p o}\end{array}\right.$

Donde:

$\mathrm{G}_{\mathrm{po}}=$ Media geométrica con presupuesto oficial. $\mathrm{V}_{\mathrm{i}}^{\mathrm{p}}=$ Valor total de cada una de las ofertas $\mathrm{i}$, sin decimales

$\mathrm{i}=$ Número de oferta

\section{Menor valor (MV)}

En este caso se selecciona la oferta de menor valor. Para la asignación de puntos, se considera la cercanía de las ofertas a dicha propuesta de menor valor, empleando la ec. (8) [4]:

$$
\text { Puntaje } i=\left\{\frac{150 *\left(V_{\text {min }}\right)}{V_{i}}\right.
$$

Donde:

$\mathrm{V}_{\text {Min }}=$ Menor valor de las ofertas válidas.

$\mathrm{V}_{\mathrm{i}}=$ Valor total sin decimales de cada una de las ofertas $\mathrm{i}$ $\mathrm{i}=$ Número de oferta.

5. Precio base $(\mathrm{PB})$

En esta fórmula se emplean los valores aritméticos de las ofertas que cumplieron los requisitos habilitantes, en la ec. (9) [4]:

$$
P B=\frac{\left(\frac{\sum P_{i}}{N_{i}}+P O\right)}{2}
$$

Donde:

$\mathrm{PB}=$ Precio Base

$\mathrm{Pi}=$ Valor de las propuestas

$\mathrm{PO}=$ Presupuesto Oficial

$\mathrm{NI}=$ Número de propuestas

Para la aplicación de esta fórmula se tienen en cuenta, además, los siguientes requisitos:

- La calificación más alta se otorga a la propuesta que presente un valor igual o inmediatamente inferior al del precio base

- La asignación de puntaje para las ofertas con precio inferior al precio base se estima considerando una relación lineal simple descendiente.

- Cuando las propuestas presentan un valor mayor al precio base, la repartición del puntaje se hace aplicando una regla de tres simple e inversa, con referencia al mayor puntaje posible para este parámetro.

6. Precio razonable (PR)

En este caso, para la evaluación de las propuestas se tiene en cuenta la información suministrada por el oferente y se emplea la ec. (10) [4]:

$$
P R O M=\frac{\sum_{i=1}^{n} V_{o i}}{n}
$$

Donde:

PROM=Promedio aritmético del precio de las propuestas a evaluar

$\mathrm{n}=$ Número de propuestas a evaluar

Voi=Valor precio de la oferta (i).

$\mathrm{i}=1,2,3 \ldots \ldots, \mathrm{n}$

Para la asignación del puntaje se considera la siguiente expresión ec. (11):

$$
P R O_{i}=\left\{\begin{array}{c}
500 *(1-X) \text { PARA } 0=<X=<1 \\
0 \text { PARA otro valor de } X
\end{array}\right.
$$

Donde:

$\mathrm{PRO}_{\mathrm{i}}=$ Menor valor de las ofertas válidas.

$\mathrm{X}=$ Valor absoluto (VOi/PROM)-1

$\mathrm{VOi}=$ Valor Precio de la oferta (i).

$\mathrm{i}=1,2 \ldots \ldots, \mathrm{n}$.

7. Ítems representativos (IR)

En este caso, el proponente debe evaluar un número de 
ítems representativos definidos por la entidad contratante en función de la mayor participación de estos ítems en el presupuesto oficial, y se modela con la ec. (12) [4], [13]:

$$
\text { PTni }=\left\{\begin{array}{l}
\text { POn } *(1-(2 *(x))) ; \text { Si } 0 \leq X \leq 0.03 \\
\frac{\text { POn }}{4} ; \text { Para cualquier otro valor de }(X)
\end{array}\right.
$$

Donde:

$$
X=\text { Valor absoluto }\left(\left(\frac{V R i}{V O i}\right)-1\right)
$$

POn=Puntaje asignado al ítem (n)de acuerdo con su incidencia en el presupuesto oficial

$\mathrm{VRi}=$ Valor del ítem $(\mathrm{n})$ con mayor participación respecto al presupuesto oficial, en la propuesta $\mathrm{i}$

$\mathrm{V} 0=$ Valor del ítem (n) con mayor participación en el presupuesto oficial

$\mathrm{i}=$ Número de propuestas a evaluar

$\mathrm{n}=$ Número de ítems a evaluar

\section{B. Análisis de la aplicación de las fórmulas en los procesos revisados}

Las distintas fórmulas se han incluido en los pliegos de condiciones, bien sea de manera individual o con diferentes combinaciones. La Tabla III resume las tipologías de combinaciones de fórmulas empleadas en los proyectos revisados.

En la investigación se encontró que el 52\% de los pliegos revisados establece solamente una para selección de las propuestas, el $22 \%$ define dos s y el $26 \%$ señala más de dos s para la selección de propuestas.

En los casos en los que hay más de una, el criterio de selección para definir la fórmula a emplear está relacionado con un rango de valores establecidos según la tasa representativa del mercado (T.R.M.), el día en que se hace entrega de las propuestas; es decir, como ejemplo, para el caso de la licitación Pública correspondiente al Proceso Número LP-002-2015 (2015), en donde la evaluación de la oferta económica podía hacerse por media aritmética o media geométrica, se seleccionó media aritmética porque al momento de la entrega de la propuesta los dos últimos dígitos de la T.R.M. estaban entre 00 y 50. Para los procesos de selección en donde intervienen más $\mathrm{s}$, se establecen otros rangos de criterio en función de la TRM.

Ahora, según la información revisada, se encontró que un $42 \%$ de las propuestas se evaluó finalmente con la Media aritmética, $30 \%$ con la Media geométrica con presupuesto oficial, $14 \%$ con la Media aritmética Alta, $6 \%$ con el criterio de Menor valor, $4 \%$ con el de Precio base y 4\% con el de Precio Razonable.

Tan solo el $2 \%$ de las propuestas incluyeron el criterio del Ítem representativo.

Por otro lado, en algunos proyectos la calificación del precio de la oferta aportaba hasta un $85 \%$ en la calificación final de la propuesta económica, frente a otros proyectos con tan solo $15 \%$. En la muestra seleccionada no se encontró una tendencia que justificara la asignación de estos porcentajes, ya que por ejemplo, en casos en donde el porcentaje sobre la propuesta económica era el 80\% se encontraron montos de \$359.984.632 (Proceso Número LP-003-2013, 2013) y de \$ 2.109.927.653 (Proceso Número LP-DO-SRN-053-2014, 2014) pero en otro proyecto con un porcentaje de $85 \%$ la cuantía fue de $\$$ 1.565.786.877 (Proceso Número 4151.LP.03.2014 [11], 2014).

$\mathrm{Al}$ respecto, es importante acotar que el puntaje asignado a este parámetro es potestad y criterio de cada entidad contratante, de acuerdo con la percepción y estudios previos que tenga del contrato a ofrecer y que, en la medida en que se incrementa el puntaje asignado a la de evaluación, se aprecia una mayor incidencia en la calificación final de los requisitos puntuables de los eventos licitatorios [5].

Otro de los aspectos analizados estuvo relacionado con los procesos de contratación en donde los proponentes se presentaron en diferentes modalidades (persona natural, persona jurídica, consorcio, unión temporal) (Tabla IV).

En cuanto a la modalidad de presentación, prevaleció el Consorcio con un peso del $61.40 \%$, muy probablemente justificado por la necesidad de cumplimiento de requisitos de experiencia en obras similares.

Se observa también que las modalidades de Unión Temporal y Persona Jurídica tuvieron una participación de $7.02 \%$ cada una, frente al $14 \%$ de licitaciones en las cuales participaron personas naturales.

Ahora, de los pliegos encontrados se observó que solamente en cuatro proyectos se presentó más de un proponente, es decir, que únicamente en un $7.02 \%$ de los eventos licitatorios la de evaluación fue un factor diferenciador en la calificación final de la oferta [5] (Tabla V).

En cuanto al oferente favorecido en los distintos contratos, se encontraron 34 consorcios, una Unión Temporal, 13 trece personas naturales y dos personas jurídicas.

$\mathrm{Al}$ respecto, se detectó que en varios de los procesos que fueron adjudicados a consorcios, se encontraban vinculadas personas naturales que obtuvieron contratos propios.

Atendiendo a los artículos señalados en la Ley Estatutaria 1581 de 2012 sobre manejo de datos personales, y con el fin de evitar interpretaciones erradas a los hallazgos de este estudio, en la Tabla V se detallan solamente los procesos en los cuales interviene más de dos veces una persona natural, bien sea actuando en nombre propio o participando en uniones temporales o consorcios; asimismo, se incluyen los montos totales de los contratos suscritos sin considerar el porcentaje de participación.

\section{Conclusiones}

Los pliegos de condiciones establecen requisitos habilitantes como son la capacidad jurídica, financiera, organizacional, acreditación de experiencia y personal. En esencia, la definición de los criterios contenidos en cada uno de estos requisitos es discrecional del equipo formulador de la propuesta.

Ahora bien, con relación a las s para la evaluación y selección de la propuesta económica, un total de siete $\mathrm{s}$ diferentes fueron identificadas, siendo las más empleadas la media aritmética y la media geométrica; y, la selección de la fórmula a aplicar, en su mayoría, dependió de los últimos 
dos dígitos de la Tasa Representativa del Mercado vigente durante el día de cierre del proceso licitatorio; de esta forma, aparentemente se libran posibles vicios en la evaluación de los proponentes.
Por otro lado, se identificaron varias personas naturales a quienes les fueron adjudicados proyectos en la franja de tiempo estudiada, así como también, se encontró que tan solo en un $7.02 \%$ de los eventos licitatorios, la de evaluación fue un factor diferenciador en la calificación final de la oferta.

TABLA III

FóRMULAS DE EVALUACIÓN SELECCIONADAS PARA CADA PROCESO

\begin{tabular}{|c|c|c|c|c|c|}
\hline Fórmulas de Evaluación Disponibles & $\begin{array}{c}\text { Puntaje } \\
\text { otorgado a la } \\
\text { fórmula }\end{array}$ & $\begin{array}{c}\text { Fórmula de } \\
\text { evaluación } \\
\text { seleccionada }\end{array}$ & $\begin{array}{l}\text { \% de la fórmula } \\
\text { respecto al } \\
\text { porcentaje total }\end{array}$ & Cuantía & Fuente \\
\hline MA & 800 & MA & $44 \%$ & $\$ 4.500 .000 .000$ & {$[16]$} \\
\hline MA & 90 & MA & $60 \%$ & $\$ 1.086 .734 .443$ & [26] \\
\hline MA & 400 & MA & $40 \%$ & $\$ 450.000 .000$ & [30] \\
\hline MA & 500 & MA & $50 \%$ & $\$ 579.151 .621$ & {$[52]$} \\
\hline MA & 750 & MA & $75 \%$ & $\$ 617.637 .874$ & [57] \\
\hline MA & 500 & MA & $22 \%$ & $\$ 743.959 .711$ & {$[58]$} \\
\hline MA, MAA, MGPO, MV & 150 & MA & $15 \%$ & $\$ 1.772 .779 .639$ & [20] \\
\hline MA, MAA, MGPO, MV & 800 & MA & $80 \%$ & $\$ 3.700 .000 .000$ & [27] \\
\hline MA, MAA, MGPO, MV & 750 & MA & $75 \%$ & $\$ 1.554 .502 .862$ & [32] \\
\hline MA, MAA, MGPO, MV & 150 & MA & $15 \%$ & $\$ 2.329 .674 .725$ & {$[55]$} \\
\hline MA, MGPO & 700 & MA & $64 \%$ & \$ 18.290.579.097 & [18] \\
\hline MA, MGPO & 600 & MA & $60 \%$ & $\$ 3.643 .703 .704$ & {$[36]$} \\
\hline MA, MGPO & 600 & MA & $60 \%$ & $\$ 1.792 .259 .748$ & {$[38]$} \\
\hline MA, MGPO & 500 & MA & $50 \%$ & $\$ 1.079 .965 .434$ & {$[44]$} \\
\hline MA, MGPO & 500 & MA & $50 \%$ & $\$ 1.683 .134 .022$ & {$[45]$} \\
\hline MA, MGPO & 400 & MA & $40 \%$ & $\$ 532.835 .238$ & {$[51]$} \\
\hline MA, MGPO & 400 & MA & $40 \%$ & $\$ 552.619 .100$ & {$[53]$} \\
\hline MA, MGPO & 400 & MA & $40 \%$ & $\$ 385.214 .827$ & {$[54]$} \\
\hline MA, MGPO & 600 & MA & $60 \%$ & $\$ 445.996 .714$ & {$[46]$} \\
\hline MA, IR, MGPO & 300 & MA & $30 \%$ & $\$ 1.308 .411 .215$ & [34] \\
\hline MA, MAA, MGPO, MV & 150 & MAA & $15 \%$ & $\$ 1.554 .502 .862$ & {$[10]$} \\
\hline MA, MAA, MGPO, MV & 750 & MAA & $75 \%$ & $\$ 2,680,438,758$ & {$[35]$} \\
\hline MAA & 800 & MAA & $80 \%$ & $\$ 359.984 .632$ & {$[47]$} \\
\hline MAA & 400 & MAA & $40 \%$ & $\$ 448.436 .039$ & {$[48]$} \\
\hline MAA & 300 & MAA & $30 \%$ & $\$ 445.767 .270$ & {$[49]$} \\
\hline MAA & 150 & MAA & $15 \%$ & $\$ 693.020 .067$ & {$[50]$} \\
\hline MAA & 800 & MAA & $80 \%$ & $\$ 2.109 .927 .653$ & {$[40]$} \\
\hline MA, MAA, MGPO, MV & 150 & MGPO & $15 \%$ & $\$ 700.934 .580$ & {$[21]$} \\
\hline MA, MGPO & 600 & MGPO & $60 \%$ & $\$ 1.095 .887 .890$ & [39] \\
\hline MA, MGPO, MV & 850 & MGPO & $85 \%$ & $\$ 1.565 .786 .877$ & {$[11]$} \\
\hline MGPO & 400 & MGPO & $40 \%$ & $\$ 1.745 .494 .235$ & {$[12]$} \\
\hline MGPO & 50 & MGPO & $50 \%$ & $\$ 1.339 .212 .820$ & {$[14]$} \\
\hline MGPO & 50 & MGPO & $50 \%$ & $\$ 732.710 .931$ & {$[15]$} \\
\hline MGPO & 400 & MGPO & $40 \%$ & $\$ 7.200 .000 .000$ & {$[17]$} \\
\hline MGPO & 600 & MGPO & $60 \%$ & $\$ 3.954 .293 .712$ & [23] \\
\hline MGPO & 600 & MGPO & $60 \%$ & $\$ 1.790 .969 .484$ & {$[41]$} \\
\hline MGPO & 500 & MGPO & $40 \%$ & $\$ 1.799 .949 .422$ & {$[43]$} \\
\hline MGPO & 700 & MGPO & $70 \%$ & $\$ 1.800 .000 .000$ & [9] \\
\hline 3.1 & 800 & MGPO & $80 \%$ & $\$ 350.137 .118$ & {$[56]$} \\
\hline MA, MAA, MGPO, MV & 150 & MGPO. & $15 \%$ & $\$ 925.925 .926$ & [19] \\
\hline MGPO. & 800 & MGPO. & $80 \%$ & $\$ 400.000 .000$ & {$[29]$} \\
\hline MGPO. & 700 & MGPO. & $70 \%$ & $\$ 1.785 .700 .000$ & {$[31]$} \\
\hline MA & 150 & MV & $15 \%$ & $\$ 5.449 .286 .552$ & {$[28]$} \\
\hline MA, MAA, MGPO, MV & 150 & MV & $15 \%$ & $\$ 676.181 .491$ & [33] \\
\hline MA, MAA, MGPO, MV & 150 & MV & $15 \%$ & $\$ 700.934 .580$ & {$[59]$} \\
\hline $\mathrm{PB}$ & 500 & $\mathrm{~PB}$ & $50 \%$ & $\$ 26.761 .079 .728$ & {$[22]$} \\
\hline PB & 500 & $\mathrm{~PB}$ & $30 \%$ & $\$ 2.429 .906 .542$ & {$[37]$} \\
\hline $\mathrm{PB}$ & 100 & $\mathrm{~PB}$ & $50 \%$ & $\$ 1.315 .484 .714$ & {$[42]$} \\
\hline PR & 500 & $\mathrm{PR}$ & $22 \%$ & $\$ 720.000 .000$ & [24] \\
\hline PR & 500 & PR & $24 \%$ & $\$ 570.960 .695$ & {$[25]$} \\
\hline
\end{tabular}


TABLA IV

MODALIDADES DE PRESENTACIÓN DE LOS PROPONENTES

\begin{tabular}{|l|l|}
\hline \multicolumn{1}{|c|}{ Modalidad } & \multicolumn{1}{c|}{ Fuente } \\
\hline Consorcio & {$[9],[10],[14],[16-23],[28],[30-37],[39-41],[44-46],[48-50],[53],[55],[58],[59]$} \\
Unión Temporal & {$[12],[15,[19],[40]$} \\
Persona Natural & {$[11],[24-27],[29],[31],[38],[40],[42],[43],[47],[54],[56]$} \\
Persona Jurídica & {$[42],[51],[52],[54]$} \\
\hline
\end{tabular}

TABLA V

PROCESOS EN DONDE INTERVIENEN MÁS DE UNA VeZ, PERSONAS NATURALES CON DIFERENTES ROLES Y PORCENTAJES DE PARTICIPACIÓN EN LOS PROYECTOS

\begin{tabular}{|c|c|c|}
\hline Proceso & Cuantía total de los contratos & Fuente \\
\hline $\begin{array}{l}\text { LP-SMIT-011-2014 en Santiago de Cali, LP-SMIT-011-2014 en Roldanillo y } \\
\text { Proceso Número } 4151 . \text { LP.03.2014 en Santiago de Cali }\end{array}$ & $\$ 4.674 .792 .601$ & {$[10-11],[32]$} \\
\hline $\begin{array}{l}\text { Proceso Número LP-SI-005-2015 en Yumbo, Proceso Número MV- } \\
\text { OBRA-005 DE } 2013 \text { en Vijes y Proceso Número LP-SMIT-013-2014 en } \\
\text { Sevilla }\end{array}$ & $\$ 32.762 .985 .380$ & {$[22],[28],[53]$} \\
\hline $\begin{array}{l}\text { Proceso Número CONV 001-2014 en Candelaria, Proceso Número } \\
\text { LP-SMIT-004-2015 en Guadalajara de Buga, Proceso Número LP- } \\
\text { SMIT-004-2015 en Florida }\end{array}$ & $\$ 1.972 .829 .855$ & {$[21],[25],[59]$} \\
\hline $\begin{array}{l}\text { Proceso Número LP-01-2014 en Dagua, Proceso Número LP-003-2013 } \\
\text { en Ginebra, Proceso Número CONV 001-2014 en Candelaria, Proceso } \\
\text { Número LP-SMIT-004-2015 en Guadalajara de Buga, Proceso Número LP- } \\
\text { SMIT-004-2015 en Florida }\end{array}$ & $\$ 2.782 .814 .487$ & {$[21],[25],[30],[47],[59]$} \\
\hline $\begin{array}{l}\text { LP-SI-005-2013 en Santiago de Cali, Proceso Número MP-SDRU- } \\
\text { LP-06-2014 en Palmira y Proceso Número } 0058 \text { en Dagua }\end{array}$ & $\$ 9.400 .000 .000$ & [9], [17], [29] \\
\hline $\begin{array}{l}\text { Proceso Número LP-SI-005-2015 y Proceso Número LP-SI-010-2013 en } \\
\text { Yumbo, Proceso Número } 188 \text { en Sevilla, Proceso Número 003-2014 en } \\
\text { Calima, LP-SI-005-2013 en Santiago de Cali, Proceso Número MP-SDRU- } \\
\text { DTI-LP-07-2015 en Palmira }\end{array}$ & $\$ 42.506 .342 .924$ & {$[9],[16],[22],[23],[27],[41]$} \\
\hline $\begin{array}{l}\text { Proceso Número 003-2014 en Candelaria, Proceso Número LP- } \\
\text { SMIT-005-2014 en Trujillo, Proceso Número 2015-160 en Caicedonia, } \\
\text { Proceso Número LP - 001-2014 en Argelia }\end{array}$ & $\$ 4.949 .855 .964$ & [24], [35], [37], [43] \\
\hline
\end{tabular}

\section{REFERENCIAS}

[1] Serrano Guzmán, M. F., Pérez Ruíz, D. D., Zambrano Díaz, N. A y Jaramillo Gómez, M. A., "Veeduría de los proyectos de infraestructura pública: una responsabilidad de todo ciudadano", Revista Educación y Humanismo, vol. 18, n 31, pp. 302-312, 2016.

[2] Cujía Guerra, E., "Desarrollo y región: Un enfoque dinámico para la superación de las desigualdades sociales", Revista Educación y Humanismo, vol. 15, nº 25, pp. 105-115, 2013.

[3] Bonometti, P., y Ruiz Seisdedos, S., "La democracia en América Latina y la constante amenaza de la desigualdad", Andamios, vol. 7, n ${ }^{\circ} 13$, pp. 11-36, mayo-agosto 2010. [4] Colombia Compra Eficiente, «www.colombiacompra.gov.co,» 19 enero 2017. [En línea]. Disponible: www.colombiacompra.gov.co.

[5] Zambrano Díaz, N. A., y Jaramillo Gómez, M. A., "Análisis de los requerimientos técnicos, financieros, organizacionales y de experiencia en proyectos de infraestructura vial: caso Valle del Cauca 2010-2015", Trabajo de grado para optar al título de Maestría en Ingeniería Civil, Pontificia Universidad Javeriana, Cali, 2016.

[6] Serrano Guzmán, M. F., Pérez Ruíz, D. D., Zambrano Díaz, N. A. y Jaramillo Gómez, M. A., "Análisis de la contratación estatal en proyectos de infraestructura vial: Caso Valle del Cauca 2010-2015", Revista Virtual Universidad Católica del Norte, (en revisión), 2017.

[7] Departamento Administrativo Nacional de Estadística, "Población por Departamento según Censo año 2005”, Bogotá, 2016.

[8] Colombia Compra Eficiente, «www.colombiacompra.gov.co, Manual de Contratación, Bogotá, 2014.

[9] Proceso Número LP-SI-005-2013, «Servicio Electrónico de
Contratación Pública SECOP,» Santiago de Cali, 2013.

[10] Proceso Número LP-SMIT-011-2014, «Servicio Electrónico de Contratación Pública SECOP,» Santiago de Cali, 2014.

[11] Proceso Número 4151.LP.03.2014, «Servicio Electrónico de Contratación Pública SECOP,» Santiago de Cali, 2014.

[12] Proceso Número SIV-LP-14-11, «Servicio Electrónico de Contratación Pública SECOP,» Buenaventura, 2015.

[13] Colombia Compra Eficiente, «Manual de la modalidad de selección de mínima cuantía,» Bogotá, 2011.

[14] Proceso Número SIV-LP-13-03, «Servicio Electrónico de Contratación Pública,» Buenaventura, 2013.

[15] Proceso Número SIV-LP-14-03, «Servicio Electrónico de Contratación Pública,» Buenaventura, 2014

[16] Proceso Número MP-SDRU-DTI-LP-07-2015, «Servicio Electrónico de Contratación Pública SECOP,» Palmira, 2015.

[17] Proceso Número MP-SDRU-LP-06-2014, «Servicio Electrónico de Contratación Pública SECOP,» Palmira, 2014.

[18] Proceso Número 330-015-008-003, «Servicio Electrónico de Contratación Pública SECOP,» Tuluá, 2014.

[19] Proceso Número PSLP-102-2015, «Servicio Electrónico de Contratación Pública SECOP,» Cartago, 2015.

[20] Proceso Número PSLP-100-2015, «Servicio Electrónico de Contratación Pública SECOP,» Cartago, 2015.

[21] Proceso Número LP-SMIT-004-2015, «Servicio Electrónico de Contratación Pública SECOP,» Guadalajara de Buga. Florida, 2015.

[22] Proceso Número LP-SI-005-2015, «Servicio Electrónico de Contratación Pública SECOP,» Yumbo, 2015. 
[23] Proceso Número LP-SI-010-2013, «Servicio Electrónico de Contratación Pública SECOP,» Yumbo, 2013.

[24] Proceso Número 003-2014, «Servicio Electrónico de Contratación Pública,» Candelaria, 2014.

[25] Proceso Número CONV 001-2014, «Servicio Electrónico de Contratación Pública,» Candelaria, 2014.

[26] Proceso Número LICITACION PÚBLICA No. 05 -PRADERA-2015, «Servicio Electrónico de Contratación Pública SECOP,» Pradera, 2015.

[27] Proceso Número 188, «Servicio Electrónico de Contratación Pública,» Sevilla, 2015.

[28] Proceso Número LP-SMIT-013-2014, «Servicio Electrónico de Contratación Pública SECOP,» Sevilla, 2014.

[29] Proceso Número 0058, «Servicio Electrónico de Contratación Pública SECOP,» Dagua, 2013.

[30] Proceso Número LP-01-2014, «Servicio Electrónico de Contratación Pública SECOP,» Dagua, 2014.

[31] Proceso Número LP-SI-004-2013, «Servicio Electrónico de Contratación Pública SECOP,» Roldanillo, 2013.

[32] Proceso Número LP-SMIT-011-2014, «Servicio Electrónico de Contratación Pública SECOP,» Roldanillo, 2014.

[33] Proceso Número LP-SMIT-010-2014, «Servicio Electrónico de Contratación Pública SECOP,» La Unión, 2014.

[34] Proceso Número LP-SMIT-001-2015, «Servicio Electrónico de Contratación Pública SECOP,» La Unión, 2015.

[35] Proceso Número LP-SMIT-005-2014, «Servicio Electrónico de Contratación Pública SECOP,» Trujillo, 2015.

[36] Proceso Número LP-002-2015, «Servicio Electrónico de Contratación Pública SECOP,» Obando, 2015.

[37] Proceso Número 2015-160, «Servicio Electrónico de Contratación Pública SECOP,» Caicedonia, 2015.

[38] Proceso Número 003, «Servicio Electrónico de Contratación Pública SECOP,» Caicedonia, 2013.

[39] Proceso Número LCP-228-2014, «Servicio Electrónico de Contratación Pública SECOP,» La Victoria, 2014

[40] Proceso Número LP-DO-SRN-053-2014, «Servicio Electrónico de Contratación Pública SECOP,» Ansermanuevo, 2014.

[41] Proceso Número LP-004-2013, «Servicio Electrónico de Contratación Pública SECOP,» Calima, 2013.

[42] Proceso Número LP-002-2013, «Servicio Electrónico de Contratación Pública SECOP,» Ansermanuevo, 2014.

[43] Proceso Número LP - 001-2014, «Servicio Electrónico de Contratación Pública SECOP,» Argelia, 2014.

[44] Proceso Número COP050-2013, «Servicio Electrónico de Contratación Pública SECOP,» Ulloa, 2013.

[45] Proceso Número LP-001, «Servicio Electrónico de Contratación Pública SECOP,» Bolívar, 2013.

[46] Proceso Número 057-2013, «Servicio Electrónico de Contratación Pública SECOP,» Andalucía, 2013.

[47] Proceso Número LP-003-2013, «Servicio Electrónico de Contratación Pública SECOP,» Ginebra, 2013.

[48] Proceso Número 100-18.03.03 O, «Servicio Electrónico de Contratación Pública SECOP,» Yotoco, 2013.

[49] Proceso Número 098 - 2014, «Servicio Electrónico de Contratación Pública SECOP,» La Cumbre, 2014.

[50] Proceso Número LP-SMIT-005-2015, «Servicio Electrónico de Contratación Pública SECOP,» Sevilla, Rio Frio, Calima, 2015.

[51] Proceso Número PLPEAV-001-2014, «Servicio Electrónico de Contratación Pública SECOP,» El Águila, 2014.

[52] Proceso Número LICITACION PÚBLICA 001, «Servicio Electrónico de Contratación Pública SECOP,» Alcalá, 2014.

[53] Proceso Número MV-OBRA-005, «Servicio Electrónico de Contratación Pública SECOP,» Vijes, 2013.

[54] Proceso Número PLPO-001-2013, «Servicio Electrónico de Contratación Pública SECOP,» Toro, 2013.

[55] Proceso Número LP-SMIT-007-2014, «Servicio Electrónico de Contratación Pública SECOP,» Caicedonia, Ansermanuevo, El Águila, Vijes, Toro, Versalles, 2014.

[56] Proceso Número LP-001-2013, «Servicio Electrónico de Contratación Pública SECOP,» El Cairo, 2013.

[57] Proceso Número PLPO-002-2015, «Servicio Electrónico de Contratación Pública SECOP,» Toro, 2015.
[58] Proceso Número 003-2015, «Servicio Electrónico de Contratación Pública SECOP,» Restrepo, 2015.

[59] Proceso Número LP-SMIT-004-2015, «Servicio Electrónico de Contratación Pública SECOP,» Florida.

Nikolas Augusto Zambrano Díaz, Ingeniero Civil. Magister en Ingeniería Civil con énfasis en Construcciones de la Pontificia Universidad Javeriana. Vinculado a la constructora Jaramillo Mora S.A.

María Alejandra Jaramillo Gómez, Ingeniera Civil. Magister en Ingeniería Civil con énfasis en Construcciones de la Pontificia Universidad Javeriana. Especialista en Gerencia de Construcciones. Vinculada a la constructora Jaramillo Mora S.A.

Diego Darío Pérez Ruiz. Profesor titular de la Pontificia Universidad Javeriana de Cali. Doctor en Ingeniería Civil de la Universidad de Texas en Arlington, Magister en Ingeniería de Tránsito y Transporte de la Universidad del Cauca. Magister en Ingeniería Civil en Recursos Hídricos de la Universidad de Puerto Rico Recinto Universitario de Mayagüez. Ingeniero Civil de la Universidad del Cauca. http://orcid.org/0000-00029656-2803

María Fernanda Serrano Guzmán. Profesor titular de la Pontificia Universidad Javeriana de Cali. Doctor en Ingeniería Civil y Magister en Ingeniería de la Universidad de Puerto Rico Recinto Universitario de Mayagüez. Especialista en Gerencia de Proyectos de Construcción de la Universidad Industrial de Santander. Especialista en Ingeniería Ambiental de la Universidad Pontificia Bolivariana de Bucaramanga. Ingeniera Civil de la Universidad Industrial de Santander. http://orcid.org/0000-0002-7366$6597 /$ 